\title{
Learning to Model Prosodic and Spectral Features for Non-parallel Emotive Speech Conversion
}

\author{
Sri Harsha Dumpala, Sageev Oore \\ Dalhousie University and Vector Institute, Canada
}

\begin{abstract}
Emotion conversion in speech has attracted recent attention owing to its importance in human-machine interaction and the current high quality of speech synthesis. Most existing approaches rely on parallel data, which is not available in many real-time applications. We propose a non-parallel emotion conversion approach based on the cycle generative adversarial network (cycleGAN) framework. We introduce new variants of cycleGAN that use recurrent neural networks and multi-kernel convolutional neural networks for modeling prosodic features along with spectral features for emotion conversion in speech. Subjective evaluation results show the effectiveness of our approach in converting natural speech, and also unseen synthesized speech samples to different target emotive states.
\end{abstract}

Keywords: Emotion conversion in speech, generative adversarial networks, prosodic features, spectral features, convolutional neural networks.

\section{Introduction}

Emotion conversion refers to the task of altering the emotive state (e.g., angry, happy, sad, neutral, etc.) of speech while preserving its linguistic content. Emotion conversion of speech has applications in various domains such as conversational chatbots, announcement systems, and in the entertainment industry.

Previous work on emotion conversion has involved modifying both prosodic features (such as fundamental frequency $\left(F_{0}\right)$ contour or pitch, tempo, volume, linear prediction residual, etc.) [1-6] and spectral features (such as spectral envelope, spectral sequence, linear prediction co-efficients (LPCs), etc.) [4-6]. However, the majority of these methods are rule-based approaches that focus on the former, i.e. modeling utterances spoken in different emotive states based on the differences in their prosodic features (which convey emotive characteristics less subtly than spectral features) [1-6]. In [2], prosodic features such as $F_{0}$ and power envelope were parameterized and modeled based on Fujisaki's model, whereas in [3], a target prediction model was used to modify these features. In [4], prosodic features such as pitch and tempo were modeled using Gaussian mixture models for emotive speech synthesis. In [5], a flexible analysis and synthesis toolkit to model LPCs, linear prediction residual and duration were proposed to perform emotion conversion in speech. Recently, rules to modify the prosodic features, such as $F 0$ contour, for neutral to emotive speech conversion with a controllable degree of emotion were proposed in [6].

Recent advancements in deep learning have enabled the use of supervised neural network based approaches for emotion conversion in speech [7, 8]. In [7], a sequence-to-sequence network modeled $F_{0}$ contour for neutral to emotive speech conversion by considering parallel speech utterances that are time-aligned at the syllable level. Further, generative adversarial networks (GANs) combined with variational autoencoders (VAEs) were also considered for modeling prosodic features, represented using the cross-wavelet transform, for emotion conversion using parallel data [8]. These works highlighted the importance of modeling $F_{0}$-based features for emotion conversion in speech.

sriharsha.d@dal.ca, sageev@vectorinstitute.ai

This article is (0) 2021 by author(s) as listed above. The article is licensed under a Creative Commons Attribution (CC BY 4.0) International license (https://creativecommons.org/licenses/by/4.0/legalcode), except where otherwise indicated with respect to particular material included in the article. The article should be attributed to the author(s) identified above. 
All of the above approaches [1-8] relied on parallel utterances (same utterance spoken by the same speaker in different emotive states), which limits their generalizability to new speakers and languages with no/few parallel utterances. Moreover, these parallel utterances need to be temporally aligned, which is very challenging owing to the temporal variations in speech, even for the same speaker, that occur naturally across different emotive states. In this paper, we propose an approach to perform emotion conversion in speech by exploiting the architecture of cycle generative adversarial networks (CycleGANs). Our approach requires only non-parallel data, thus removing the need for time-aligned parallel utterances.

The main contributions of this work are as follows.

- We analyze the importance of modeling both spectral and $F_{0}$ values for emotion conversion

- We propose the use of bidirectional long-short-term memory (BLSTM) layers for the generator network, and convolutional layers with multiple kernels for the discriminator network to model the $F_{0}$ values for emotion conversion in speech.

The rest of the paper is organized as follows. Related work is summarized in Section 2. A brief review of cycleGAN architecture is provided in Section 3. Our proposed approach to model $F_{0}$ contour, and spectral features for emotion conversion in speech is detailed in Section 4. Experimental details along with the evaluation results, are explained in Section 5. Summary and conclusions are given in Section 6.

\section{Related Work}

Generative adversarial networks (GANs) consist of two different networks: a generator and a discriminator. [9]. The generator and discriminator are trained jointly so that the discriminator learns to distinguish between the inputs that belong to the original training distribution from those that do not, while the generator is trained to generate examples that will fool the discriminator, i.e. that will appear as though they are from the training distribution. The idea is that as they train - effectively playing a two-player min-max game - they will each improve the other's performance.

GANs have obtained impressive results in image generation [10], image-image translation [11] and style transfer [12]. GANs were also successfully applied to speech-based applications such as speech synthesis $[13,14]$ and speech denoising $[15,16]$. Further, GANs were considered for emotion conversion in speech using parallel data where the cross-wavelet based representation of speech was modified [8]. In [17], non-parallel emotion conversion was performed by training an autoencoder combined with a discriminator (distinguishing fake samples from real samples) to learn the disentangled representation of emotion space.

CycleGANs are a variant of GANs, capable of performing translation between different domains without the need for parallel data [18-20]. CycleGANs have shown success in unpaired image-to-image translation [18]. In audio processing, they have been applied to spectrograms for unpaired timbre conversion between musical instruments [21]. In speech processing, cycleGANs were used for non-parallel data based voice conversion [22, 23], crosslingual voice conversion [24], and perturbed to normal speech conversion [25, 26]. In this work, we perform non-parallel emotive speech conversion using cycleGAN based architecture [18].

Most previous work on non-parallel emotion conversion using cycleGAN architectures $[17,27,28]$ modeled spectral features (which usually represent the vocal tract system characteristics), not prosodic ones (which represent the excitation source characteristics). In [28], a convolutional network based cycleGAN was considered for modeling only spectral features for emotion conversion. But it is evident from $[7,8,29]$ that the excitation source is significantly affected compared to the vocal tract system during the production of different emotions in speech. The spectral and prosodic features are complementary and ought 
to both be considered for emotion conversion. Thus, in this work we modify both the $F_{0}$ contour, associated with the excitation source characteristics, along with the spectral envelope, associated with the vocal tract system characteristics. Recently [30, 31], convolutional network based cycleGANs were considered for modeling the $F_{0}$ values. In [30], a convolutional network based cycleGAN was considered for modeling the $F_{0}$ values represented using a continuous wavelet transform (CWT) for emotion conversion. In [31], the generator network consisted of a convolutional neural network followed by a deterministic block with static parameters for modeling the F0 contour. We introduce auto-regressive generator networks to better model the multi-resolution temporal coherence of the $F_{0}$ contour.

To the best of our knowledge, all the previous works using GANs for speech processing have only considered convolutional layers for the generator and the discriminator networks $[14,17,22,25]$. But the emotion-specific information in the prosodic features is embedded in the temporal relationships existing in the $F_{0}$ contour, at both sub-segmental and suprasegmental levels. To capture these temporal relationships, we propose the use of recurrent neural networks (RNNs). We therefore introduce the use of BLSTM layers for the generator network, and convolutional layers with multiple kernels for the discriminator network. In this paper, we will model both spectral and prosodic features to convert neutral speech to the target emotion.

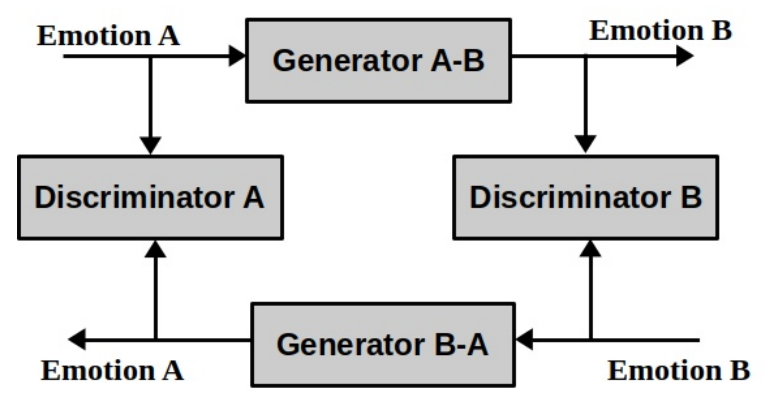

Figure 1. Block diagram depicting the outline of CycleGAN architecture considered for emotion conversion in speech. Emotion A and Emotion B refer to the source and the target emotions, respectively.

\section{CycleGAN Architecture for Emotion Conversion}

A brief review of the cycleGAN architecture for emotion conversion in speech is provided in this section. Let $\mathrm{E}_{A}$ represent the set of (possible) utterances having emotion label $A$, and $\mathrm{E}_{B}$ represent the set of utterances having emotion label $B$. So for example, $e_{A} \in E_{A}$ is an example utterance, and the examples in the training set having associated emotion $A$ constitute a subset of $E_{A}$, i.e. $\mathcal{T}_{A} \subset E_{A}$. Note that $\mathcal{T}_{A}$ and $\mathcal{T}_{B}$ need not be parallel, i.e. our system does not assume that the training set includes the utterances having the same words while spoken in different emotive states.

As shown in Figure 1, the cycleGAN architecture consists of a pair of generator networks i.e., Generator A-B $\left(\mathrm{G}_{A-B}\right)$ and Generator B-A $\left(\mathrm{G}_{B-A}\right)$, and a pair of discriminator networks i.e., Discriminator A $\left(\mathrm{D}_{A}\right)$ and Discriminator B $\left(\mathrm{D}_{B}\right) . \mathrm{G}_{A-B}$ learns a mapping such that $G_{A-B}\left(e_{A}\right) \in E_{B}$, i.e. any utterance $e_{A}$ with emotion $A$ is mapped to a parallel utterance (i.e. saying the same words) with emotion $B$. Similarly, $\mathrm{G}_{B-A}$ learns a mapping such that $G_{B-A}\left(e_{B}\right) \in E_{A}$, i.e., any utterance $e_{B}$ with emotion $B$ is mapped to a parallel utterance with emotion $A . \mathrm{D}_{A}$ discriminates real source samples $\mathrm{e}_{A}$ from the translated 


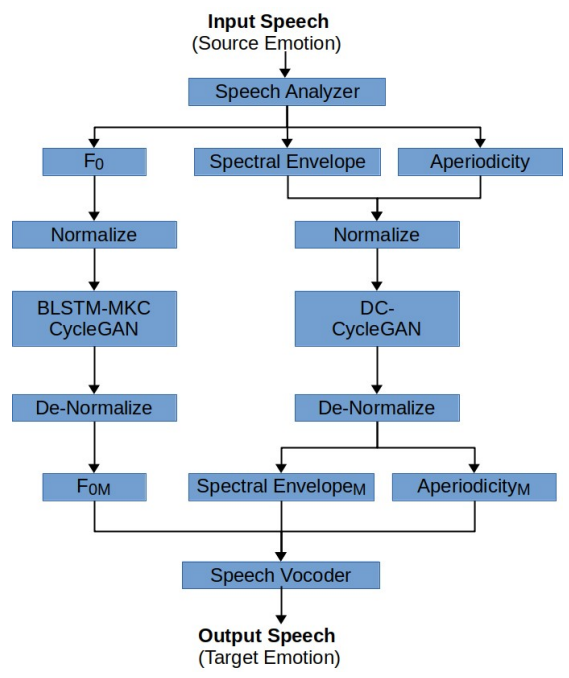

Figure 2. Block diagram of proposed approach. $F_{0 M}$, Spectral Envelope $_{M}$ and Aperiodicity $_{M}$ refers to the modified $F_{0}$, Spectral Envelope and Aperiodicity, respectively.

samples $\mathrm{G}_{B-A}\left(\mathrm{e}_{B}\right)$. Similarly, $\mathrm{D}_{B}$ discriminates real target samples $\mathrm{e}_{B}$ from the translated samples $\mathrm{G}_{A-B}\left(\mathrm{e}_{A}\right)$.

The different loss functions considered in the training of cycleGAN models are:

- Adversarial loss: This loss is used for matching the distribution of the generated samples to the distribution of the target domain samples.

The adversarial loss corresponding to $\mathrm{G}_{A-B}$ and $\mathrm{D}_{B}$ is:

$$
\begin{aligned}
\mathcal{L}_{a d v}\left(G_{A-B}, D_{B}\right) & =\mathbb{E}_{e_{B}}\left[\log \left(D_{B}\left(e_{B}\right)\right)\right] \\
& +\mathbb{E}_{e_{A}}\left[\log \left(1-D_{B}\left(G_{A-B}\left(e_{A}\right)\right)\right)\right],
\end{aligned}
$$

where $G_{A-B}$ tries to generate samples $G_{A-B}\left(e_{A}\right)$ that are similar to the samples from the target domain $\mathrm{E}_{B}$, while $D_{B}$ tries to distinguish between translated samples $\left(G_{A-B}\left(e_{A}\right)\right)$ and the real samples $\mathrm{E}_{B}$.

Similarly, the adversarial loss corresponding to $\mathrm{G}_{B-A}$ and $\mathrm{D}_{A}$ is:

$$
\begin{aligned}
\mathcal{L}_{a d v}\left(G_{B-A}, D_{A}\right) & =\mathbb{E}_{e_{A}}\left[\log \left(D_{A}\left(e_{A}\right)\right)\right] \\
& +\mathbb{E}_{e_{B}}\left[\log \left(1-D_{A}\left(G_{B-A}\left(e_{B}\right)\right)\right)\right],
\end{aligned}
$$

- Cycle-consistent loss: Training the generators $\left(\mathrm{G}_{A-B}\right.$ and $\left.\mathrm{G}_{B-A}\right)$ using only adversarial loss does not guarantee that the generators will be able to preserve the content of the input samples while modifying only the domain related part of the inputs. To encourage $\mathrm{G}_{A-B}$ and $\mathrm{G}_{B-A}$ to find $\left(\mathrm{e}_{A}, \mathrm{e}_{B}\right)$ pairs with the same contextual information, reconstruction or cycle-consistent loss is introduced as follows:

$$
\begin{aligned}
\mathcal{L}_{c y c}\left(G_{A-B}, G_{B-A}\right) & =\mathbb{E}_{e_{A}}\left[\left\|e_{A}-G_{B-A}\left(G_{A-B}\left(e_{A}\right)\right)\right\|_{1}\right] \\
& +\mathbb{E}_{e_{B}}\left[\left\|e_{B}-G_{A-B}\left(G_{B-A}\left(e_{B}\right)\right)\right\|_{1}\right],
\end{aligned}
$$

where the translated sample $\mathrm{G}_{A-B}\left(e_{A}\right)$ is provided as input back to the generator $\mathrm{G}_{B-A}$, and $\mathrm{G}_{B-A}$ tries to reconstruct back $\mathrm{e}_{A}$. Similarly, the translated sample $\mathrm{G}_{B-A}\left(e_{B}\right)$ is provided as input back to the generator $\mathrm{G}_{A-B}$, and $\mathrm{G}_{A-B}$ tries to 


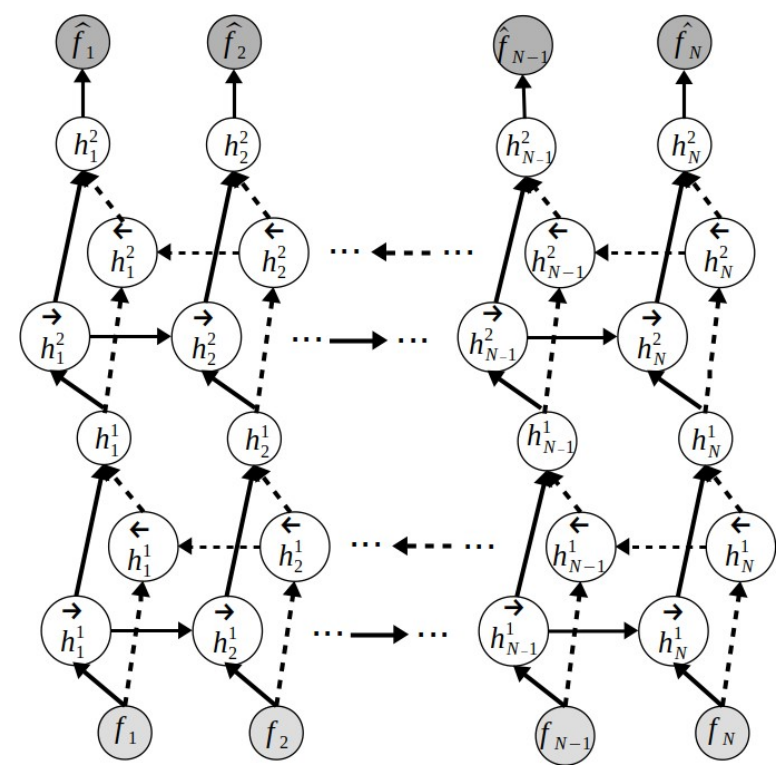

Figure 3. Block diagram of generator with bi-directional LSTM (BLSTM) layers. $\left\{f_{1}\right.$, $\left.f_{2}, \cdots f_{N-1}, f_{N}\right\},\left\{\hat{f}_{1}, \hat{f}_{2}, \cdots \hat{f}_{N-1}, \hat{f}_{N}\right\}$ represent the source and target $F_{0}$ contours, respectively. $h^{1}, h^{2}$ refers to the first and second BLSTM layer parameters, respectively.

reconstruct back $\mathrm{e}_{B}$. In this work, we are using $L_{1}$ norm for the reconstruction loss, which is shown to be more effective in non-parallel speech conversion tasks [22].

- Identity loss: To encourage generators to learn a mapping such that the linguistic information is preserved, identity loss is introduced as follows:

$$
\begin{aligned}
\mathcal{L}_{i d}\left(G_{A-B}, G_{B-A}\right) & \left.=\mathbb{E}_{e_{B}}\left[\| e_{B}-G_{A-B}\left(e_{B}\right)\right) \|_{1}\right] \\
& +\mathbb{E}_{e_{A}}\left[\left\|e_{A}-G_{B-A}\left(e_{A}\right)\right\|_{1}\right] .
\end{aligned}
$$

The identity loss function encourages the generator to find a mapping that preserves composition between the input and the output.

Finally, the objective function to be optimized is defined as:

$$
\begin{aligned}
\mathcal{L}=\lambda_{a d v} \mathcal{L}_{a d v}\left(G_{A-B}, D_{B}\right)+ & \lambda_{a d v} \mathcal{L}_{a d v}\left(G_{B-A}, D_{A}\right) \\
& +\lambda_{c y c} \mathcal{L}_{c y c}+\lambda_{i d} \mathcal{L}_{i d},
\end{aligned}
$$

where $\lambda_{a d v}, \lambda_{c y c}$ and $\lambda_{i d}$ are the hyper-parameters which signify the relative importance of the adversarial, cycle-consistent and identity losses, respectively.

\section{Proposed Approach}

Our proposed approach for emotion conversion is depicted in Figure 2. As shown in Figure 2, we model the $F_{0}$ contour with a BLSTM-MKC-cycleGAN: a cycleGAN with a BLSTMbased generator and multi-kernel convolution (MKC) based discriminator. We model the spectral envelope and aperiodicity parameters with a deep convolutional (DC) cycleGAN. We use the "WORLD" toolkit [32] as a speech analyzer (to extract features from speech signal) and as a vocoder (to synthesize speech from the modified/converted features). The details of the proposed system for emotion conversion are as follows. 


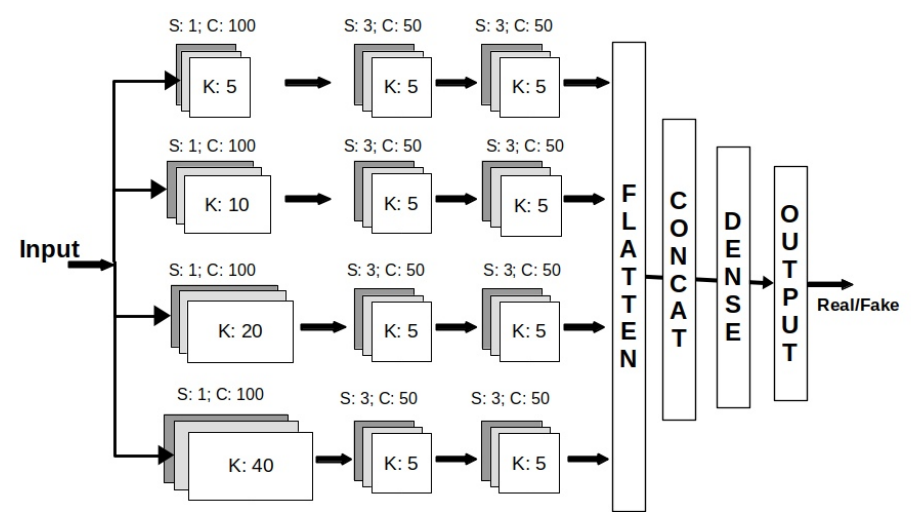

Figure 4. Block diagram of multi-kernel CNN based discriminator. Here, K, S, C refers to kernel size, stride and number of channels, respectively. Input can be spectral + aperiodic or $F_{0}$.

\subsection{Modeling $F_{0}$ contour}

To capture the important temporal relationships that exist in $F_{0}$ contours for different emotions, we propose a BLSTM-MKC-cycleGAN model: as shown in Figure 3, the generator network consists of two bi-directional LSTM (BLSTM) layers (each layer consisting of 256 units). The $F_{0}$ value of the target emotion, corresponding to each $F_{0}$ value in the source emotion, is estimated by considering the output from the second BLSTM layer at each time step.

This target $F_{0}$ contour is provided as input to a multi-kernel convolutional (MKC) based discriminator, as depicted in Figure 4. This MKC discriminator (of the BLSTM-MKCcycleGAN model) consists of three 1-D convolutional layers with each layer having four kernels of different sizes. Multiple kernels with different sizes (which were previously used in text processing $[33,34]$ ) are considered to capture the temporal relationships existing at different resolutions. The output from each kernel of the third convolutional layer are flattened and then concatenated before passing through a dense layer, and finally through an output layer. The objective of the discriminator is to classify the input $F_{0}$ contour as real/fake.

\subsection{Modeling spectral and aperiodic features}

The cycleGAN architecture we use to model spectral and aperiodic features is adapted from [25], which is a deep convolutional cycleGAN (DC-cycleGAN). As shown in Figure 5, the generator network is made up of three main blocks: namely, a down-sampling block, a residual block and an up-sampling block. The initial convolution layer ('CONV') consists of 128 filters with a kernel of size $1 \times 18$ and a stride of 1 . The down-sampling block includes two convolutional layers with 256 and 512 filters, respectively (each with a kernel of size 6 and a stride of size 2). The residual block consists of 6 convolutional layers (each layer with 1024 filters with a kernel of size 3 and stride of size 1) with residual connections. The up-sampling block consists of two convolutional layers with with 1024 and 512 filters, respectively (each with a kernel of size 6 and a stride of size $\frac{1}{2}$. The final convolution layer ('CONV') consists of 36 filters with a kernel of size $1 \times 18$, and a stride of size 1 . All the convolutional layers are 1-dimensional to preserve the temporal structure. Further, gated linear units (GLUs) [35] along with instance normalization [12] are applied to all the convolutional layers of DC-cycleGAN. 


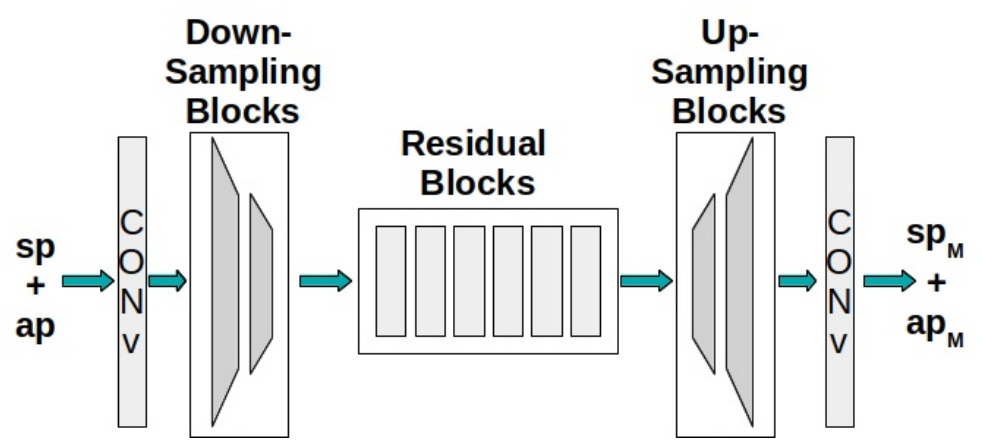

Figure 5. Block diagram of deep convolutional cycleGAN generator. Input is spectral envelope (sp) concatenated with aperiodic (ap) features. $\operatorname{sp}_{M}$ and $\operatorname{ap}_{M}$ are the modified spectral envelope and aperiodic features, respectively. Conv refers to a convolutional layer.

The discriminator of the DC-cycleGAN is also a multi-kernel convolutional network as shown in Figure 4 with certain modifications. 2-dimensional convolutional layers are considered for the discriminator of the DC-cycleGAN, whereas the convolutional layers of the discriminator used for modeling the $F_{0}$ contour are 1-dimensional. Three different kernels with sizes 3, 4 and 6 are used for the first layer. Moreover, the last two fully connected layers are discarded, and the adversarial loss (mean squared error loss) is computed directly on the concatenated output of the discriminator.

\subsection{Training details}

Both cycleGANs (BLSTM-MKC-cycleGAN for modeling $F_{0}$ and DC-cycleGAN for spectral and aperiodic feature modeling) are trained using Adam optimizer [36] with $\beta_{1}=0.5$. Initial learning rates of 0.0002 and 0.00016 are used for the generator and the discriminator networks respectively, with a decay factor of $2 \times 10^{5}$ for each iteration after the first 5000 iterations/steps. Dropout rates of 0.4 and 0.3 are used for the generator and the discriminator networks of the BLSTM-MKC-cycleGAN, and batch sizes of 8 and 2, respectively. All the cycleGAN models with deep convolutional generators are trained for 1500 epochs with an early stopping threshold of 40 epochs. All the cycleGAN models with BLSTM generators are trained for 200 epochs with an early stopping threshold of 5 epochs.

The proposed networks are trained by considering three different losses, namely, adversarial loss $\left(\mathcal{L}_{a d v}\right)$, cyclic loss $\left(\mathcal{L}_{c y c}\right)$ and identity loss $\left(\mathcal{L}_{i d}\right)$ [18, 22, 25], as explained in Section 3. Further, Gradient penalty $\left(\mathcal{L}_{g p}\right)[37,38]$ is used to stabilize the training of the cycleGANs and to improve the quality of the generated speech. As given in Eq. 3.5, these losses are scaled by hyper-parameters $\lambda_{a d v}, \lambda_{c y c}$ and $\lambda_{i d}$ to signify the relative importance of each loss. We use $\lambda_{a d v}=5, \lambda_{c y c}=10$ and $\lambda_{i d}=5$ in this work. The $\mathcal{L}_{g p}$ loss is also scaled with a trade-of-parameter $\lambda_{g p}$. In this work, we considered $\lambda_{g p}=5$.

\section{Experiments and Results}

\subsection{Dataset details}

Experiments were performed using the Interactive Emotional Dyadic Motion Capture (IEMOCAP) database [39]. This database consists of recordings of conversations between pairs of speakers (each pair chosen randomly from a set consisting of 5 female and 5 male speakers) in each session collected across 5 different sessions. All the original recordings were annotated using 7 emotion labels, of which we consider 4 for in this study: neutral, 
angry, happy and sad. We used a total of 1500 utterances (400 neutral utterances, 400 angry utterances, 350 angry utterances and 350 happy utterances) spoken by the female speakers. Each utterance is of $2-5$ seconds in duration. For each emotion class, 20 utterances per emotion were used for validation, 20 utterances per emotion were used for testing, and the remaining utterances of each emotion were used in training. It is to be noted that the utterances in different emotion classes are non-parallel.

\subsection{Feature extraction}

We use the WORLD vocoder system [32] to extract features (i.e., $F_{0}$, spectral and aperiodic) from the raw speech signals sampled at $16 \mathrm{KHz}$. We extract 35 -dimensional Mel filter bank (MFB), 1-dimensional aperiodic (AP) and 1-dimensional $F_{0}$ values from the speech signal within a window of length 20 -msec for every 5 -msec. We perform mean-variance normalization of the feature vectors, where the mean and variance values are computed for each emotion class separately over the training samples corresponding to that class.

During training, we randomly select 160 contiguous feature frames from each sample in the train set, and repeat this process for every epoch. During testing, we provide all the feature frames extracted from the speech sample as input to the system, and the output of the system is the converted feature frames. Given the predicted spectral aperiodic and $F_{0}$ features, we used the WORLD vocoder to synthesize speech.

\subsection{Evaluation procedure}

Listening tests were performed on the converted speech to evaluate the performance of the proposed emotion conversion system. The converted speech samples were evaluated based on voice quality and perceived emotion state. All 10 neutral test utterances were converted into the three target emotions i.e., angry, sad and happy. A total of 15 subjects participated in the listening tests, and each utterance was annotated by at least 5 human listeners in terms of voice quality and perceived emotion state. For annotating the voice quality, the listeners were asked to give an opinion score on a scale of 5 ( 5 for the best and 0 for the worst). For annotating the perceived emotion state, the listeners were asked to provide a confidence score on a scale of 5 ( 5 , if closer to target emotion and 0 , if closer to source emotion) based on the source and the target emotions.

We compared the performance of four systems: first, the two baselines consisted of (1) a cycleGAN trained only with spectral features (CGAN-S), and (2) a convolutional-based cycleGAN trained on both spectral and prosodic features (ConvGAN-SP) [30]. We also included (3) our proposed network without gradient penalty (Prop), and (4) a variation of our proposed network that did include gradient penalty loss (Prop-GP). Note that the gradient penalty loss [37]-which we have shown provides a consistent improvement in this context-has not been previously considered in cycleGAN-based emotional speech conversion systems [30].

\subsection{Results}

The mean opinion score (MOS) for voice quality of different systems is shown in Figure 6. It can be observed that the voice quality of the proposed network (Prop) is comparable to the baseline systems. It can also be observed that including the gradient penalty loss further improves the perceived voice quality of the converted speech.

The MOS obtained for perceived emotion state is shown in Figure 7; it can be observed from this that modeling both spectral and prosodic features results in better perceived emotion conversion compared to modeling only spectral features. Further, our proposed network performs better than a convolution layer based cycleGAN for prosodic feature 

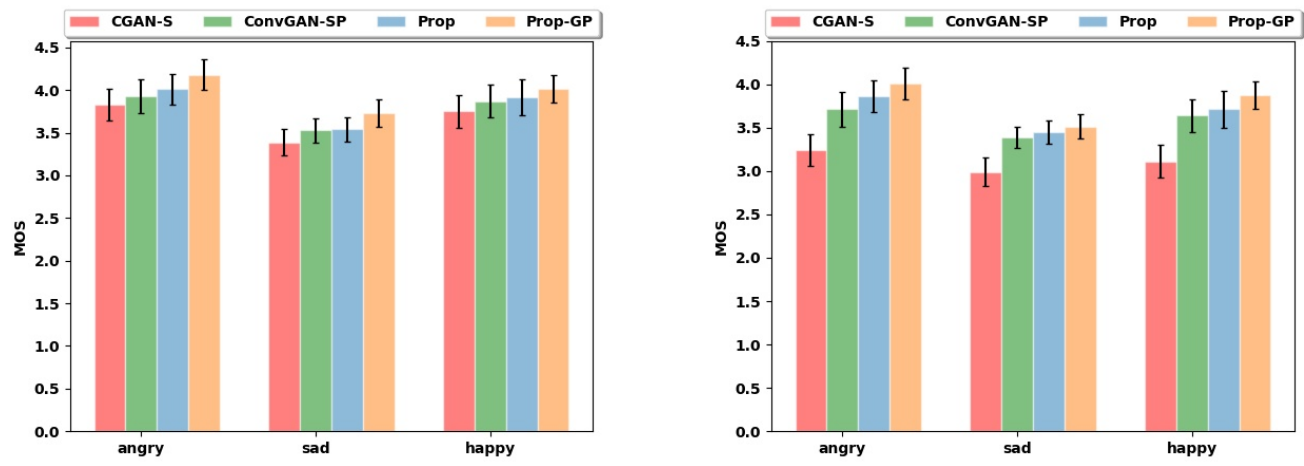

Figure 6. Mean opinion score (MOS) for voice quality of different systems. Angry, sad and happy refer to the converted target emotions from neutral speech.

Figure 7. Mean opinion score (MOS) for perceived emotion state of different systems. Angry, sad and happy refer to the converted target emotions.

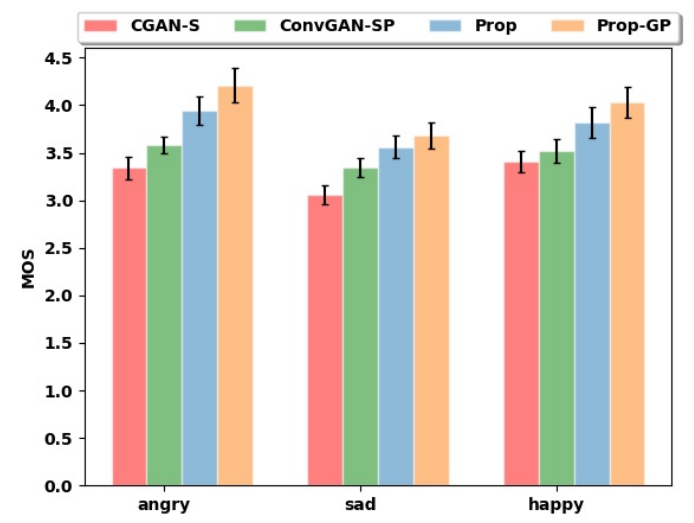

Figure 8. Mean opinion score (MOS) for perceived emotion state of different systems. Angry, sad and happy refer to the converted target emotions when synthesized speech samples are provided as input.

conversion. This could be because of the auto-regressive capability of the BLSTM network, and the ability of the multi-kernel CNN based discriminator to better identify the temporal variations existing in the $F_{0}$ contour across different emotional states. It is also evident from Figure 7 that the proposed network trained with the gradient penalty loss (Prop-GP) has a better ability to convert speech across different emotions compared to other systems. While our system appears to be perceptually better in every condition, it is noticeably more so for conversion into certain emotions in particular, such as conversion from neutral to anger.

To analyze the emotion conversion ability of the proposed approach on synthesized speech, we considered speech generated using a pre-trained tacotron model [40]. We converted the synthesized speech, which is in neutral voice, to different emotive states, and performed subjective evaluation. Note that all models are trained only on natural speech samples; synthesized speech is never seen by the models during the training phase.

The MOS obtained for different emotion conversion systems is shown in Figure 8. It can be observed from Figure 8 that the proposed networks (both Prop and Prop-GP) are 
Table 1. Mean opinion score (MOS) obtained for the perceived emotion state of PropGP and Sup-RNN systems. Angry, sad and happy refer to the converted target emotions from neutral speech

\begin{tabular}{|l|c|c|}
\hline Emotion & Sup-RNN & Prop-GP \\
\hline Neutral-angry & 2.69 & 4.18 \\
\hline Neutral-sad & 2.45 & 3.57 \\
\hline Neutral-happy & 2.58 & 3.89 \\
\hline
\end{tabular}

perceptually more successful at converting synthesized speech into different emotive states when compared to other emotion conversion systems.

To analyze the importance of cycleGANs over the conventional supervised recurrent neural network (Sup-RNN) model, proposed in [7], for modeling $F_{0}$ contour for emotion conversion, we trained the Sup-RNN model with the same non-parallel training data used to train the cycleGAN models. Note that the adversarial, cycle-consistent, identity and gradient-penalty loss functions were used for training the cycleGAN models whereas negative log-likelihood loss function was used to train the Sup-RNN model in a supervised manner. The MOS obtained for the proposed system with gradient penalty (Prop-GP) and supervised RNN-based system (Sup-RNN) for emotion conversion are shown in Table 1. For both systems, the spectral and aperiodic features are converted using the ConvGAN-SP system. It can be observed (Table 1) that the proposed emotion conversion system (Prop-GP) outperforms the supervised RNN-based system (Sup-RNN) for $F_{0}$ conversion.

When the Sup-RNN model is trained using non-parallel data (as considered in this work), we observed that the predicted $F_{0}$ values are constant throughout the utterances i.e., a flat $F_{0}$ contour is generated. This shows the significance of cycleGAN-based approaches when compared to the conventional approach (as in [7]).

\section{Summary and Conclusion}

In this paper, we proposed the use of a BLSTM-based generator and multi-kernel CNN based discriminator networks in cycleGAN architecture for non-parallel emotional speech conversion. Subjective evaluations show that our proposed approach is perceptually better than other non-parallel emotion conversion systems in terms of voice quality and emotion conversion ability. Further, our proposed network can effectively convert even synthesized speech into different emotive states.

\section{Acknowledgements}

The authors would like to thank Rudolf Uher and his group for discussions. The authors also thank the anonymous reviewers for their valuable feedback which helped improve the quality of the paper. Resources used in preparing this research were provided, in part, by NSERC, the Province of Ontario, the Government of Canada through CIFAR, and companies sponsoring the Vector Institute www.vectorinstitute.ai/\#partners.

\section{References}

[1] M. Wang, M. Wen, K. Hirose, and N. Minematsu. "Emotional voice conversion for mandarin using tone nucleus model-small corpus and high efficiency". In: Speech Prosody. 2012.

[2] H Fujisaki and K Hirose. "Analysis of voice fundamental frequency contours for declarative sentences of Japanese". In: Journal of the Acoustical Society of Japan (E) 5.4 (1984), pp. 233242. 
[3] Y. Xue and M. Akagi. "A study on applying target prediction model to parameterize power envelope of emotional speech". In: NCSP Workshop. 2016.

[4] Z. Wang and Y. Yu. "Multi-level prosody and spectrum conversion for emotional speech synthesis". In: ICSP. 2014, pp. 588-593.

[5] P Gangamohan, V. K. Mittal, and B. Yegnanarayana. "A Flexible Analysis Synthesis Tool (FAST) for studying the characteristic features of emotion in speech". In: 2012 IEEE Consumer Communications and Networking Conference (CCNC). IEEE. 2012, pp. 250-254.

[6] Y. Xue, Y. Hamada, and M. Akagi. "Voice conversion for emotional speech: Rule-based synthesis with degree of emotion controllable in dimensional space". In: Speech Communication 102 (2018), pp. 54-67.

[7] C. Robinson, N. Obin, and A. Roebel. "Sequence-to-sequence Modelling of F0 for Speech Emotion Conversion". In: ICASSP. IEEE. 2019, pp. 6830-6834.

[8] Z. Luo, J. Chen, T. Takiguchi, and Y. Ariki. "Neutral-to-emotional voice conversion with cross-wavelet transform F0 using generative adversarial networks". In: APSIPA Transactions on Signal and Information Processing 8 (2019).

[9] I. Goodfellow, J. Pouget-Abadie, M. Mirza, B. Xu, D. Warde-Farley, S. Ozair, A. Courville, and Y. Bengio. "Generative adversarial nets". In: NIPS. 2014.

[10] E. L. Denton et al. "Deep generative image models using a laplacian pyramid of adversarial networks". In: NIPS. 2015.

[11] P. Isola, J.-Y. Zhu, T. Zhou, and A. A. Efros. "Image-to-Image Translation with Conditional Adversarial Networks". In: CVPR. 2017.

[12] J. Johnson, A. Alahi, and L. Fei-Fei. "Perceptual losses for real-time style transfer and superresolution". In: ECCV. Springer. 2016.

[13] Y. Saito, S. Takamichi, and H. Saruwatari. "Statistical parametric speech synthesis incorporating generative adversarial networks". In: IEEE/ACM Trans. on Audio, Speech, and Lang. Proc. 26.1 (2017), pp. 84-96.

[14] A. Marafioti, N. Holighaus, N. Perraudin, and P. Majdak. "Adversarial generation of timefrequency features with application in audio synthesis". In: preprint arXiv:1902.04072 (2019).

[15] S. Pascual, A. Bonafonte, and J. Serra. "SEGAN: Speech enhancement generative adversarial network". In: arXiv preprint arXiv:1703.09452 (2017).

[16] C. Donahue, B. Li, and R. Prabhavalkar. "Exploring speech enhancement with generative adversarial networks for robust speech recognition". In: ICASSP. IEEE. 2018, pp. 5024-5028.

[17] J. Gao, D. Chakraborty, H. Tembine, and O. Olaleye. "Nonparallel Emotional Speech Conversion". In: INTERSPEECH. 2019, pp. 2858-2862.

[18] J. Zhu, T. Park, P. Isola, and A. A. Efros. "Unpaired image-to-image translation using cycleconsistent adversarial networks". In: arXiv preprint (2017).

[19] Z. Yi, H. Z., P. Tan, and M. Gong. "DualGAN: Unsupervised Dual Learning for Image-toImage Translation." In: ICCV. 2017.

[20] T. Kim, M. Cha, H. Kim, J. K. Lee, and J. Kim. "Learning to Discover Cross-Domain Relations with Generative Adversarial Networks". In: ICML. 2017.

[21] S. Huang, Q. Li, C. Anil, X. Bao, S. Oore, and R. B. Grosse. "TimbreTron: A WaveNet (CycleGAN(CQT(Audio))) Pipeline for Musical Timbre Transfer". In: (2019).

[22] T. Kaneko and H. Kameoka. "Parallel-data-free voice conversion using cycle-consistent adversarial networks". In: preprint arXiv:1711.11293 (2017).

[23] T. Kaneko, H. Kameoka, K. Tanaka, and N. Hojo. "Cyclegan-vc2: Improved cyclegan-based non-parallel voice conversion". In: ICASSP. IEEE. 2019, pp. 6820-6824.

[24] Y Zhao, W. Huang, X. Tian, J. Yamagishi, R. K. Das, T. Kinnunen, Z. Ling, and T. Toda. "Voice Conversion Challenge 2020: Intra-lingual semi-parallel and cross-lingual voice conversion". In: arXiv preprint arXiv:2008.12527 (2020).

[25] S. H. Dumpala, I. Sheikh, R. Chakraborty, and S. K. Kopparapu. "Cycle-consistent gan frontend to improve asr robustness to perturbed speech". In: NeurIPS IRASL workshop (2018).

[26] S. H. Dumpala, I. Sheikh, R. Chakraborty, and S. K. Kopparapu. "A Cycle-GAN approach to model natural perturbations in speech for ASR applications". In: arXiv preprint arXiv:1912.11151 (2019). 
[27] D. Wan, H. Dong-Yan, L. Danqing, and Z. Yuexian. "Speech Emotion Recognition using Spectral Normalized CycleGAN". In: ACIIW Workshops and Demos. IEEE. 2019, pp. 93-99.

[28] T. Asakura, S. Akama, E. Shimokawara, T. Yamaguchi, and S. Yamamoto. "Emotional Speech Generator by using Generative Adversarial Networks". In: ISICT. 2019, pp. 9-14.

[29] P Gangamohan, S. R. Kadiri, and B. Yegnanarayana. "Analysis of emotional speech at subsegmental level." In: INTERSPEECH. 2013, pp. 1916-1920.

[30] K. Zhou, B. Sisman, and H. Li. "Transforming Spectrum and Prosody for Emotional Voice Conversion with Non-Parallel Training Data". In: arXiv preprint arXiv:2002.00198 (2020).

[31] R. Shankar, J. Sager, and A. Venkataraman. "Non-Parallel Emotion Conversion Using a DeepGenerative Hybrid Network and an Adversarial Pair Discriminator". In: Proc. Interspeech 2020 (2020), pp. 3396-3400.

[32] M. Morise, F. Yokomori, and K. Ozawa. "WORLD: a vocoder-based high-quality speech synthesis system for real-time applications". In: IEICE Trans. on Information and Systems 99.7 (2016).

[33] S. Poria, E. Cambria, D. Hazarika, N. Majumder, A. Zadeh, and L. Morency. "Contextdependent sentiment analysis in user-generated videos". In: ACL. 2017, pp. 873-883.

[34] I. Sheikh, S. H. Dumpala, R. Chakraborty, and S. K. Kopparapu. "Sentiment analysis using imperfect views from spoken language and acoustic modalities". In: $H M L$-Challenge, $A C L$. 2018, pp. 35-39.

[35] Y. N. Dauphin, A. Fan, M. Auli, and D. Grangier. "Language Modeling with Gated Convolutional Networks". In: ICML. 2017.

[36] D. P Kingma and J. Ba. "Adam: A method for stochastic optimization". In: arXiv preprint arXiv:1412.6980 (2014).

[37] I. Gulrajani, F. Ahmed, M. Arjovsky, V. Dumoulin, and A. C. Courville. "Improved training of wasserstein gans". In: NIPS. 2017, pp. 5767-5777.

[38] H. Thanh-Tung, S. Venkatesh, and T. Tran. "Improving generalization and stability of generative adversarial networks". In: ICLR 2019: Proceedings of the 7th International Conference on Learning Representations. ICLR. 2019.

[39] C. Busso, M. Bulut, C. Lee, A. Kazemzadeh, E. Mower, S. Kim, J. N. Chang, S. Lee, and S. S. Narayanan. "Iemocap: Interactive Emotional Dyadic Motion Capture database, Language Resources and Evaluation". In: (2008).

[40] Y. Wang, R. Skerry-Ryan, D. Stanton, Y. Wu, R. J. Weiss, N. Jaitly, Z. Yang, Y. Xiao, Z. Chen, S. Bengio, et al. "Tacotron: Towards End-to-End Speech Synthesis". In: Interspeech (2017), pp. 4006-4010. 